\title{
Hamartomatous Tumors in the Gastrointestinal Tract
}

\author{
Estelle Cauchin Yann Touchefeu Tamara Matysiak-Budnik \\ IMAD, Hepato-Gastroenterology and Digestive Oncology, Hôtel Dieu/CHU de Nantes, \\ Nantes, France
}

\section{Key Words}

Cowden syndrome · Genetic predisposition · Hamartomatous polyposis · Hamartomatous polyps · Juvenile polyposis · Peutz-Jeghers syndrome

\begin{abstract}
Background: Digestive hamartomatous polyps are a rare entity. They may be sporadic (solitary Peutz-Jeghers polyp or solitary juvenile polyp) or reveal genetic predisposition like Peutz-Jeghers syndrome, juvenile polyposis or Cowden disease. Summary: Diagnosis is based on personal and family history and on clinical data including physical signs (in particular dermatological), endoscopic findings (the number of polyps) and histological features of the polyps. The risk of complications and of digestive and extra-digestive cancers may be high, especially in case of genetic predisposition syndromes, and requires multidisciplinary management of the patients (oncogenetic counseling, gastroenterologist, pathologist, dermatologist, gynecologist and endocrinologist). Endoscopic evaluation is very helpful to establish the current situation, to perform polypectomy and to allow for good histological examination of the polyps, whose degeneration has been exceptionally described. The recent development of new molecular techniques (next-generation DNA sequencing) allows for rapid multiple gene sequencing and facilitates diagnosis. Key Message: Discovery of a hamartomatous polyp requires a rigorous work-up which should be performed by a multidisciplinary team, including a genetic oncologist, experienced in this pathology. Practical Implications: The diagnostic procedure in hamartomatous polyps should be based on the number of polyps identified during endoscopy (solitary versus multiple) and on their histological characteristics. The clinical examination must search for mucosal and skin lesions. If a polyposis syndrome is identified, oncogenetic consultation is necessary in order to define screening modalities for family members, aiming at preventing cancer development. Endoscopic resection (polypectomy) of the lesions may prevent complications like bleeding and degeneration and also diminish the risk of surgery and subsequent short bowel syndrome.




\section{Introduction}

Digestive hamartomatous polyps are a rare entity. They may be solitary (solitary PeutzJeghers (PJ) polyp or solitary juvenile polyp) or multiple, the latter often associated with genetic predisposition usually inherited in an autosomal dominant manner, like PJ syndrome (PJS), juvenile polyposis (JP), Cowden disease and other PTEN hamartoma tumor syndromes, or syndromes of unknown origin like Cronkhite-Canada syndrome. Diagnosis is based on personal and family history and on clinical data obtained during physical examination (in particular dermatological), endoscopy (specifying the number and localization of polyps) and histological analysis of the polyps. The two major issues associated with hamartomatous polyps are, on one hand, the risk of related complications (gastrointestinal bleeding, obstruction, infarction, invagination, etc.), frequently responsible for marked morbidity and sometimes mortality, and, on the other hand, the increased risk of cancer, especially in the genetic forms of the disease. Indeed, the risk of digestive and extra-digestive cancers related to genetic hamartomatous polyposis syndromes may be high and thus requires multidisciplinary management of these patients (oncogenetic counseling, gastroenterologist, pathologist, dermatologist, gynecologist and endocrinologist) and a specific surveillance strategy. Endoscopic observation is very helpful to evaluate the phenotype of the disease and to perform a polypectomy, as well as to allow for good histological examination of these polyps. The progress in the understanding of the genetic and molecular mechanisms involved in the genesis of these tumors allows to better understand their associations with cancer development and thus to improve surveillance, but also to hope for the development of targeted therapies in the future. The aim of this review is to describe the different hamartomatous polyp entities, with emphasis on practical points related to the clinical management of patients.

\section{Diagnosis of Hamartomatous Polyps and Syndromes}

The suspicion of a hamartomatous polyp or of hamartomatous polyposis syndromes imposes a rigorous diagnostic procedure. The diagnosis is usually based on clinical symptoms and may be sometimes difficult due to a great diversity of clinical pictures. Information about medical history is important and should be focused on a personal history of intestinal invagination in childhood or a family history of polyposis. Clinical examination should search for macrocephaly, goiter or bone abnormalities (bone cysts). Dermatological evaluation may provide important information, e.g. mucocutaneous pigmentation with buccal lentiginosis, peribuccal pigmentation and pigmentation on a dorsal face of hands and feet which may orientate towards the diagnosis of PJS, as well as presence of telangiectasia and nevi are in favor of JP, whereas the presence of facial papules, oral papillomatosis, acral keratosis, genital pigmentation, lipomas or fibromas is rather characteristic for Cowden syndrome.

The role of histology is crucial. In adults, hamartomatous polyps of the digestive tract are rare as compared to adenomatous or hyperplastic polyps. They are composed of normal cellular elements but have a markedly distorted architecture. Histological diagnosis of hamartomatous polyps is relatively easy, but it is more difficult to specify the particular type of polyp. Indeed, different types of hamartomatous polyps may be distinguished, such as PJ polyps, hamartomatous polyps of juvenile type, hamartomatous polyps related to ganglioneuromatosis (Cowden syndrome) and hamartomatous polyps of Cronkhite-Canada type.

PJ polyps are usually multilobular and have a very characteristic histology, showing mucosa with interdigitating smooth muscle bundles that yield a characteristic branching tree appearance termed arborization. They may be easily confounded with serrated polyps or 
hyperplastic polyps. Juvenile polyps have a spherical appearance with short pedicles and are microscopically characterized by an overgrowth of edematous lamina propria with inflammatory cells and cystic glands. They may sometimes take the form of raisin grapes and harbor dysplasia. Some adenomas have been described in the proximity of juvenile polyps. The distinction between a juvenile polyp and an inflammatory polyp may be sometimes difficult.

The polyps found in ganglioneuromatosis like Cowden disease develop from the connective tissue of the submucosa. Their histology may be diverse and may correspond to connective tissue pseudopolyps, lymphoid or lipomatous polyps or adenomatous polyps. Some exceptional cases of degeneration have been described.

The polyps observed in Cronkhite-Canada syndrome are characterized by cystic dilatation of the mucosal glands, massive submucosal edema and infiltration by inflammatory cells.

\section{Sporadic Hamartomatous Polyps}

Two types of solitary polyps may be distinguished: PJ-type polyps and juvenile-type solitary polyps.

\section{Peutz-Jeghers-Type Solitary Polyps}

PJ-type solitary polyps are rare and usually observed during the 4th decade of life. The circumstances of their discovery are various; patients may be totally asymptomatic or present with vague abdominal pain, dyspepsia or digestive bleeding. In cases reported in the literature, these polyps did not seem to be associated with extra-digestive manifestations such as skin lesions or with a family history of PJS [1]. From a histological point of view, solitary PJ polyps are identical to those present in PJ polyposis syndrome but without other phenotypic characteristics. Initial colonoscopy associated with upper endoscopy and exploration of the small intestine are usually recommended [1]. Polypectomy allows for histological analysis of the polyps but may also prevent the risk of degeneration exceptionally reported in this type of polyps. Some authors recommend systematic search for the deleterious STK11/LKB1 gene mutation, even in the absence of clinical diagnostic criteria of PJS [1]. The long-term evolution is not well documented and there is no established recommendation for screening [2].

\section{Juvenile Sporadic Polyps}

Juvenile sporadic polyps are the most common gastrointestinal polyps found in children. Their incidence is estimated at $2 \%$ in children under the age of 10 . They are usually situated in the rectosigmoid region and have to be distinguished from JP syndrome. Some cases of adenomas and colorectal adenocarcinomas associated with sporadic juvenile polyps have been reported [3].

\section{Hamartomatous Polyposis Syndromes (fig. 1)}

The presence of multiple polyps, together with characteristic clinical signs and a family history of polyposis, should always lead to the suspicion of hamartomatous polyposis related to genetic predisposition and to oncogenetic consultation.

\section{Peutz-Jeghers Syndrome}

PJS is a syndrome of genetic predisposition to colorectal cancer transmitted in an autosomal dominant manner. The incidence of PJS is low, estimated between 1/8,300 and $1 / 200,000$ births [4]. The diagnosis can be suspected in case of acute intestinal invagination 


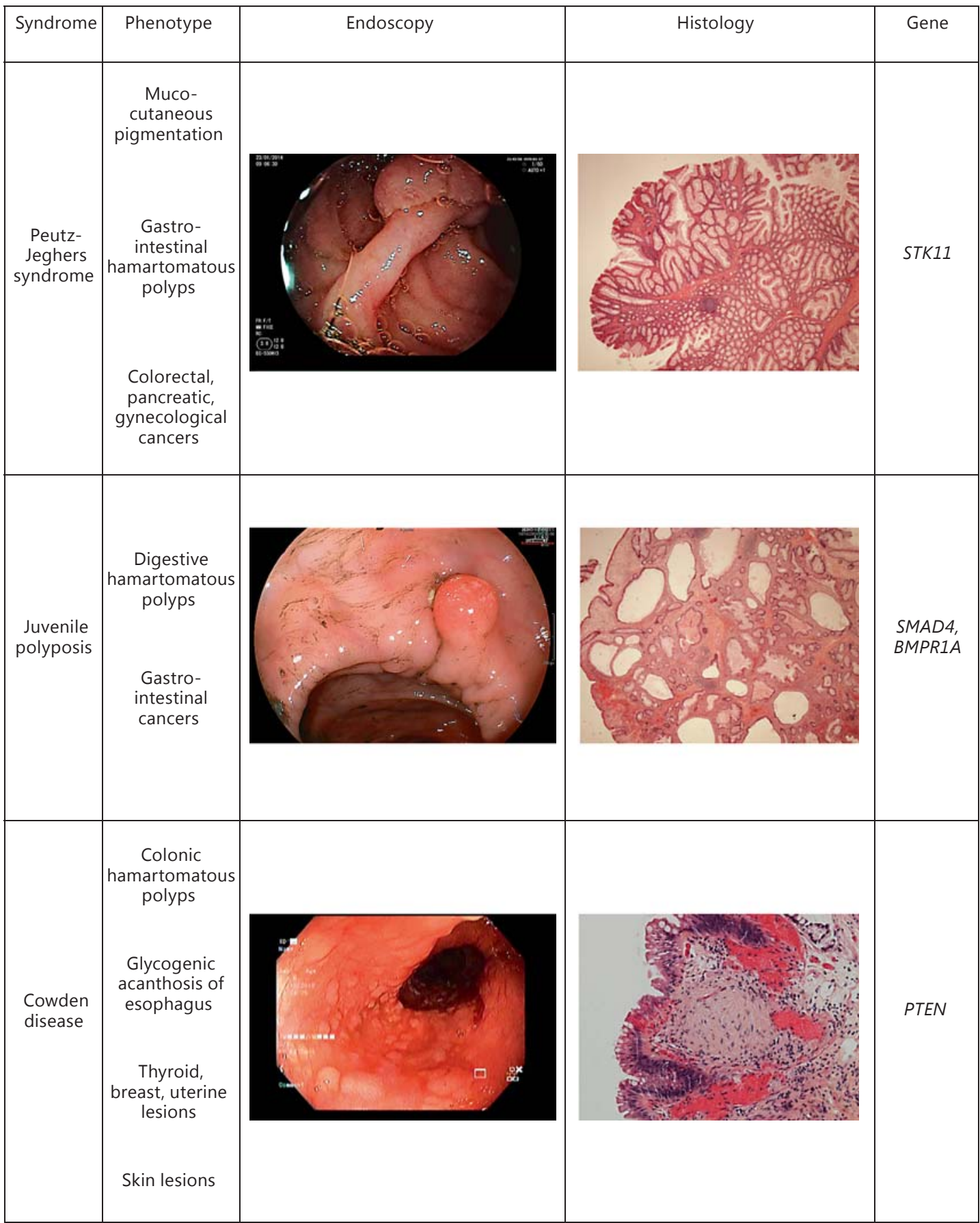

Fig. 1. Genetic hamartomatous polyposis syndromes: clinical, endoscopic and histological features.

in a child, digestive bleeding or anal polyp emission. The most typical clinical presentation is abdominal pain secondary to acute intestinal invagination. In one third of cases the diagnosis is made during the first decade of life, at the occasion of an exploration of digestive bleeding, anemia or abdominal pain related to intestinal infarction, occlusion or invagination. In $60 \%$ of cases the diagnosis is made later, during the 2 nd or 3 rd decade. 
PJS is defined by clinical criteria comprising characteristic mucocutaneous lesions present in $95 \%$ of cases, which usually appear in childhood and attenuate after puberty, and by the presence of hamartomatous polyps of characteristic histology, mainly situated in the small intestine, but also in the colon.

The diagnosis of PJS may be made in the presence of one of the following criteria: one or more PJS polyps, one or more PJS polyps detected in a subject with a family history of PJS, characteristic mucocutaneous pigmentation in a subject with a family history of PJS, or one or more PJS polyps detected in a subject with characteristic mucocutaneous lesions.

According to the studied population and the techniques used, the deleterious mutation which occurs in STK11 gene localized on chromosome 19 (19p13) is identified in 30-80\% of cases. In the study by Aretz et al. [5] including 56 patients, if one of the criteria was fulfilled, the deleterious STK11 gene mutation was identified in $94 \%$ of cases (64\% point mutation, $30 \%$ deletions).

The risk of digestive (colorectal, gastric or pancreatic) and extra-digestive (breast, ovarian, uterine, testicular, gonadoblastoma) cancers in PJS is highly increased. In an Italian multicenter study including 119 patients with PJS, the overall relative risk of cancer of any localization was close to 15 , the risk of cervical cancer was $>55$, and the risk of pancreatic cancer was $>139$ [6]. The ANAES 2004 guidelines recommend realization of a first colonoscopy at the age of 18 , and then every $2-3$ years [7].

The following surveillance modalities can be proposed according to Beggs et al. [8]: (1) Annual clinical examination. (2) Annual full blood count and liver function test. (3) Baseline upper endoscopy and colonoscopy at the age of 8 years. If normal, the following endoscopy can be proposed at the age of 18, and then every 3 years or less if there are alarm symptoms. If polyps are found, surveillance endoscopy every 3 years until the age of 50 and then every 1-2 years should be performed. (4) Video capsule endoscopy of the small intestine every 3 years starting from the age of 8. (5) Breast self-examination starting from the age of 18. (6) Annual breast MRI from the age of 25-50, thereafter annual mammography. (7) Annual examination and testicular examination from birth until 12 years, completed by ultrasound if abnormalities are observed. (8) Cervicovaginal smears with cytologic examination three times yearly starting from the age of 25 .

For pancreatic surveillance, no clear benefit of systematic screening for pancreatic cancer has been demonstrated until now. Some authors propose pancreatic ultrasound every 1-2 years starting from the age of 25-30 [3].

Video capsule endoscopy and double balloon enteroscopy play an important role in the surveillance of PJS. Removal of polyps by polypectomy is essential for the prevention of complications such as bleeding, anemia, invagination or bowel obstruction. It also allows reduction of the risk of surgery and subsequent short bowel syndrome. Accordingly, some authors propose endoscopic resection of all polyps $>15 \mathrm{~mm}$, even in asymptomatic patients [9].

Medical treatments like mTOR inhibitors, Cox2 inhibitors, metformin or aromatase inhibitors are currently under evaluation, but are not part of clinical routine yet.

\section{Juvenile Polyposis}

JP is a rare entity, also transmitted in a dominant autosomal manner, whose incidence is estimated at 1/100,000 births per year [10]. Its histological diagnosis is difficult. The penetrance of JP is incomplete and heterogeneous, with a family history present only in $20-50 \%$ of cases [9].

Three categories of JP have been described: colonic JP, pediatric JP characterized by diarrhea, exudative enteropathy and digestive hemorrhage, and generalized JP. The diagnosis is usually made during the first or second decade of life [11] during the exploration of rectorrhagia, invagination, prolapse or diarrhea related to exudative enteropathy. 
The majority of polyps are localized in the colon (rectosigmoid region), in the stomach and more rarely in the duodenum, jejunum or ileum [12]. The number of polyps may be very high, from 50 to 200 , but there is some variability between families and between different members within the same family. Adenomas have also been associated with juvenile polyps. Extra-digestive abnormalities are sometimes observed, like skin (telangiectasia, nevi), bone (hypertelorism, bone cysts), cerebral (hydrocephalus) or cardiovascular (interventricular communications or arteriovenous malformations typical for Rendu-Osler disease) anomalies.

The diagnosis of JP is based on one of the following criteria: (1) Presence of at least three juvenile polyps associated with gastrointestinal diffuse polyposis. (2) Presence of one or more juvenile polyps in a subject belonging to a family bearing this syndrome.

JP shows a great genetic heterogeneity. Mutations of the SMAD4 gene situated on chromosome 18 (18q21) or of the BMPR1A gene situated on chromosome 10 (10q22-23) are identified only in $60 \%$ of typical forms. Other genetic forms exist, and they implicate both PTEN and BMPR1A genes. These forms are usually severe, associated with diffuse polyposis and exudative enteropathy and responsible for premature death. A higher frequency of gastric polyposis and gastric cancers has been reported in case of SMAD4 gene mutations than in case of BMPR1A gene mutations [10,13].

The risk of gastric and colorectal cancer in JP is increased, and for the latter it is estimated to be 34 times higher compared to the general population at a mean age of 44 years [14]. The risk of colon cancer is probably secondary to the presence of foci of dysplasia within the juvenile polyps and to the presence of frequently associated adenomas. The risk of pancreatic and duodenal cancer is weaker [15].

The ANAES 2004 guidelines propose the first colonoscopy at the age of 10-15 [7]. If the result is normal (absence of polyps), a follow-up colonoscopy may be proposed after 3 years [16]. If the result is abnormal (one or more polyps present), two options exist: if the number of polyps is small $(<20)$, iterative polypectomies are recommended, but if the polyps characteristics justify it (high number, accelerated growth, bleeding), prophylactic surgery can be considered $[12,15,16]$. Curative surgery may also be proposed in case of dysplastic or neoplastic lesions identified. In case of dysplasia or metaplasia, upper endoscopy with gastric mucosal cartography should be performed before surgical decision $[12,16]$.

\section{Cowden Syndrome}

This genetic syndrome of predisposition to cancer is transmitted as an autosomal dominant disease whose incidence is estimated at $1 / 200,000$ individuals. Its intra- and interfamily penetrance is variable.

The diagnosis of Cowden syndrome is essentially clinical and requires a complete clinical examination (dermatological, mammary, neurological, cervical, abdominal). The precise diagnostic criteria (pathognomonic, major and minor) have been established by the International Cowden Consortium [17] (fig. 2).

Mucocutaneous lesions (98\%), macrocephaly (93\%), gastrointestinal polyps (93\%) and breast $(74 \%)$ and thyroid (71\%) benign lesions are the most typical signs of this syndrome [18]. Digestive lesions are observed in $70-85 \%$ of patients. They may appear early and are usually asymptomatic; they are present within the whole digestive tract (colon, rectum, stomach, duodenum) and are frequently associated with hyperplastic polyps, ganglioneuromatous or adenomatous polyps [19]. Glycogenic acanthosis of the esophagus, visible after Lugol staining, is often observed. Other clinical signs may be observed, such as inconstantly present vascular abnormalities and urogenital anomalies (ovarian cysts, fibroma or uterine leiomyoma). 
Table 1. Management program for men and women with Cowden syndrome from the National Comprehensive Cancer Network (adapted from Jelsig et al. [22])

Women

Breast awareness starting at the age of 18 years

Clinical breast examination every 6-12 months, starting at the age of 25 years or 5-10 years before the earliest known breast cancer in the family

Annual mammography and breast MRI screening starting at the age of 30-35 years or individualized based on earliest age of onset in the family

For endometrial cancer screening, encourage patient education and prompt response to symptoms and participation in clinical trials to determine the effectiveness and necessity of screening modalities

Discuss risk-reducing mastectomy and hysterectomy and counsel regarding degree of protection, extent of cancer risk and reconstruction options

Address psychological, social and quality of life aspects of undergoing risk-reducing mastectomy and/or hysterectomy

Men and women

Annual comprehensive physical examination starting at the age of 18 years or 5 years before the youngest age of diagnosis of a component cancer in the family (whichever comes first), with particular attention to breast and thyroid examination

Annual thyroid examination starting at the age of 18 years or 5-10 years before the earliest known thyroid cancer in the family, whichever is earlier

Colonoscopy starting at the age of 35 years, then every 5 years or more frequently if the patient is symptomatic or polyps are found

Consider renal ultrasound starting at the age of 40 years, then every $1-2$ years

Dermatological management may be indicated for some patients

Consider psychomotor assessment in children at diagnosis and brain MRI if there are symptoms

Education regarding the signs and symptoms of cancer

\section{PTEN Hamartoma Tumor Syndrome}

The group of PTEN hamartoma tumor syndrome corresponds to the clinical entity embracing different syndromes characterized by the presence of constitutional mutation in the PTEN gene. Besides the already described JP and Cowden disease, it comprises BannayanRiley-Ruvalcaba syndrome, Lhermitte-Duclos disease, SOLAMEN syndrome and macrocephaly-autism syndrome, all of them considered as variants of Cowden disease. BannayanRiley-Ruvalcaba syndrome associates macrocephaly, development retardation, lipomatosis, vascular malformations, intestinal hamartomatous polyps and genital masculine pigmentation. Lhermitte-Duclos syndrome is characterized by abnormal development and enlargement of the cerebellum, while SOLAMEN syndrome is characterized by segmentary disproportionate development, lipomatosis, arteriovenous malformations and nevi.

\section{Cronkhite-Canada Syndrome}

Cronkhite-Canada syndrome is rare, of unknown etiology, characterized by the presence of diffuse gastrointestinal polyposis classically sparing the esophagus, associated with alopecia, skin pigmentation and nail atrophy. There are no genetic tests allowing detection of this syndrome in individuals with suspected clinical features. It seems to be an acquired 
affection, responsible for multiple deficiencies in iron, magnesium and calcium. The prognosis is poor with a 5-year mortality rate of $45-55 \%$. Complications include gastrointestinal bleedings, intestinal invaginations and rectal prolapse. The risk of gastric and colorectal cancer seems to be increased [16], which justifies surveillance endoscopy (upper endoscopy and colonoscopy). Some authors propose prophylactic colectomy.

Other Syndromes with Hamartomatous Polyps

Hamartomatous polyps have been described in patients with neurofibromatosis of type 1, in multiple endocrine neoplasia of type 2B, in Gorlin syndrome or in Birt-Hogg-Dubé syndrome [22].

\section{Conclusion}

Hamartomatous polyposis syndromes are rare. The value of histology and clinical examination, in particular dermatological, is crucial for orientating diagnosis. Given an increased risk of digestive and extra-digestive cancers, the multidisciplinary clinical management of these patients is highly warranted. For gastroenterologists careful endoscopic examination is necessary, with endoscopic removal of polyps, which reduces the risk of complications such as bleeding, but also degeneration and the risk of surgery.

\section{Acknowledgments}

We would like to thank Prof. Jean-François Mosnier for histology pictures and Dr. Marc Le Rhun and Dr. Nicolas Musquer for endoscopic pictures.

\section{Disclosure Statement}

The authors have no disclosure to make.

\section{References}

1 Retrosi G, Nanni L, Vecchio FM, Manzoni C, Canali R, Busato G, Pintus C: Solitary Peutz-Jeghers polyp in a paediatric patient. Case Rep Gastroenterol 2010;4:452-456.

2 Culver EL, McIntyre AS: Sporadic duodenal polyps: classification, investigation, and management. Endoscopy 2011;43:144-155.

-3 Giardiello FM, Trimbath JD: Peutz-Jeghers syndrome and management recommendations. Clin Gastroenterol Hepatol 2006;4:408-415.

4 Van Lier MG, Wagner A, Mathus-Vliegen EM, Kuipers EJ, Steyerberg EW, van Leerdam ME: High cancer risk in Peutz-Jeghers syndrome: a systematic review and surveillance recommendations. Am J Gastroenterol 2010; 105:1258-1264.

5 Aretz S, Stienen D, Uhlhaas S, Loff S, Back W, Pagenstecher C, McLeod DR, Graham GE, Mangold E, Santer R, Propping P, Friedl W: High proportion of large genomic STK11 deletions in Peutz-Jeghers syndrome. Hum Mutat 2005;26:513-519.

6 Resta N, Pierannunzio D, Lenato GM, Stella A, Capocaccia R, Bagnulo R, Lastella P, Susca FC, Bozzao C, Loconte DC, Sabbà C, Urso E, Sala P, Fornasarig M, Grammatico P, Piepoli A, Host C, Turchetti D, Viel A, Memo L, Giunti L, Stigliano V, Varesco L, Bertario L, Genuardi M, Lucci Cordisco E, Tibiletti MG, Di Gregorio C, Andriulli A, Ponz de Leon M; AIFEG: Cancer risk associated with STK11/LKB1 germline mutations in Peutz-Jeghers syndrome patients: results of an Italian multicenter study. Dig Liver Dis 2013;45:606-611.

7 Recommandations pour la pratique clinique: endoscopie digestive basse: indications en dehors du dépistage en population, ANAES, April 2004. 
-8 Beggs AD, Latchford AR, Vasen HF, Moslein G, Alonso A, Aretz S, Bertario L, Blanco I, Bülow S, Burn J, Capella G, Colas C, Friedl W, Møller P, Hes FJ, Järvinen H, Mecklin JP, Nagengast FM, Parc Y, Phillips RK, Hyer W, Ponz de Leon M, Renkonen-Sinisalo L, Sampson JR, Stormorken A, Tejpar S, Thomas HJ, Wijnen JT, Clark SK, Hodgson SV: Peutz-Jeghers syndrome: a systematic review and recommendations for management. Gut 2010;59:975986.

-9 Campos FG, Figueiredo MN, Martinez CA: Colorectal cancer risk in hamartomatous polyposis syndromes. World J Gastrointest Surg 2015; 7:25-32.

10 Calva D, Howe J: Cancer syndromes. Bethesda, National Center for Biotechnology Information, 2009.

11 Manfredi M: Hereditary hamartomatous polyposis syndromes: understanding the disease risks as children reach adulthood. Gastroenterol Hepatol 2010;6:185-196.

12 Tenière P, Songne K, Frebourg T, Moguelet P, Foulatier O, Michot F, Le Pessot F, Le Blanc I, Scotte M: Juvenile polyposis coli. The usefulness of a genetic study and the role of surgical treatment. Gastroenterol Clin Biol 2002;26:1047-1050.

-13 Aretz S, Stienen D, Uhlhaas S, Stolte M, Entius MM, Loff S, Back W, Kaufmann A, Keller KM, Blaas SH, Siebert R, Vogt S, Spranger S, Holinski-Feder E, Sunde L, Propping P, Friedl W: High proportion of large genomic deletions and a genotype update in 80 unrelated families with juvenile polyposis syndrome. J Med Genet 2007;44: 702-709.

14 Brosens LA, van Hattem A, Hylind LM, Iacobuzio-Donahue C, Romans KE, Axilbund J, Cruz-Correa M, Tersmette AC, Offerhaus GJ, Giardiello FM: Risk of colorectal cancer in juvenile polyposis. Gut 2007;56:965-967.

15 Howe JR, Mitros FA, Summers RW: The risk of gastrointestinal carcinoma in familial juvenile polyposis. Ann Surg Oncol 1998;5:751-756.

16 Calva D, Howe JR: Hamartomatous polyposis syndromes. Surg Clin North Am 2008;88:779-817.

17 Pilarski R, Burt R, Kohlman W, Pho L, Shannon KM, Swisher E: Cowden syndrome and the PTEN hamartoma tumor syndrome: systematic review and revised diagnostic criteria. J Natl Cancer Inst 2013;105:1607-1616.

-18 Bubien V, Bonnet F, Brouste V, Hoppe S, Barouk-Simonet E, David A, Edery P, Bottani A, Layet V, Caron O, Gilbert-Dussardier B, Delnatte C, Dugast C, Fricker JP, Bonneau D, Sevenet N, Longy M, Caux F; French Cowden Disease Network: High cumulative risks of cancer in patients with PTEN hamartoma tumour syndrome. J Med Genet 2013;50:255-263.

19 Heald B, Mester J, Rybicki L, Orloff MS, Burke CA, Eng C: Frequent gastrointestinal polyps and colorectal adenocarcinomas in a prospective series of PTEN mutations carriers. Gastroenterology 2010;139:1927-1933.

20 Riegert-Johnson DL, Gleeson FC, Roberts M, Tholen K, Youngborg L, Bullock M, Boardman LA: Cancer and Lhermitte-Duclos disease are common in Cowden syndrome patients. Hered Cancer Clin Pract 2010;8:6.

21 Nieuwenhuis MH, Kets CM, Murphy-Ryan M, Colas C, Möller P, Hes FJ, Hodgson SV, Olderode-Berends MJ, Aretz S, Heinimann K, Gomez Garcia EB, Douglas F, Spigelman A, Timshel S, Lindor NM, Vasen HF: Is colorectal surveillance indicated in patients with PTEN mutations? Colorectal Dis 2012;14:e562-e566.

22 Jelsig AM, Qvist N, Brusgaard K, Nielsen CB, Hansen TP, Ousager LB: Hamartomatous polyposis syndromes: a review. Orphanet J Rare Dis 2014;9:101. 